\title{
Postvaccination Acute Disseminated Encephalomyelitis and Guillain-Barré Syndrome
}

\author{
Bojan Rugole, Željka Ležaić
}

Can J Neurol Sci. 2014; 41: 389-391

Acute disseminated encephalomyelitis (ADEM) and Guillain-Barré syndrome (GBS) are monophasic inflammatory diseases of the central and peripheral nervous systems, respectively. Both ADEM and GBS are immune-mediated neurological complications mostly associated with recent infection or vaccination. ${ }^{1,2}$ According to the theory of molecular mimicry, microbial antigens can induce aberrant cross-reactive immune responses and putative targets are specific myelin components and/or oligodendrocytes. Subsequent neural damage occurs as a result of cellular and/or humoral inflammatory activation. Neurologic complications occur mostly within a few months of immunization. Postvaccination ADEM occurs in a minority of cases and is more frequent in children and adolescents. ${ }^{3}$ Although there are no pathognomonic symptoms, a combination of multifocal neurologic disturbances and altered consciousness is the main feature of the disease. The most common form of GBS following vaccination is acute inflammatory demyelinating polyneuropathy (AIDP) while acute motor axonal neuropathy (AMAN) occurs rarely. Most case reports of ADEM and GBS evolving after vaccination describe patients who developed one of these complications, rather than both simultaneously. In the case presented, patient developed paraparesis and signs of encephalopathy several weeks after influenza vaccination. Based on clinical course and findings, upon exclusion of other possible causes, the patient was diagnosed with ADEM and pure motor axonal form of GBS presumably as a consequence of influenza vaccination. To our knowledge, this may be the first reported case of co-occurrence of ADEM and AMAN following influenza vaccination.

\section{Case Report}

A 78-year-old female was referred to a neurologist because of progressive weakness of both legs, evolving over hours to days. The patientat first declined hospital treatment but several days later she was referred again because of loss of consciousness and right hand clumsiness. Her husband described episodes of unconsciousness lasting about one minute during which she appeared awake, but unaware of surroundings, exhibiting involuntary chewing and lip smacking movements. Past medical history was positive for heart attack, arterial hypertension and renal impairment. Vaccination against influenza, conducted three weeks before the first symptom appeared, was the only relevant event which preceded present illness. However, it was noted that she had also received the same vaccination once a year during the past several years, and on the last two occasions, after each vaccination she reported to her general practitioner the development of general weakness in her legs lasting several weeks. The influenza vaccine she received was a trivalent vaccine comprised of inactivated hemagglutinin surface antigens from each of the three virus strains (influenza subtypes A and B). At the time of the examination she was afebrile and normotensive. She appeared disinhibited in behaviour and disoriented in time. Neurologic examination revealed moderately decreased strength in the right arm and proximal muscles of lower extremities, accentuated deep tendon reflexes in lower limbs without pathological reflexes. All modalities of sensations were preserved and there was no sphincter dysfunction.

Initial laboratory test revealed blood urea nitrogen 9.4 $\mathrm{mmol} / \mathrm{L}$, creatinine $141 \mu \mathrm{mol} / \mathrm{L}$. Levels of erythrocyte sedimentation rate, C-reactive protein, complete blood cell count, blood glucose, electrolytes, creatine kinase, liver enzymes, and thyroid hormones were unremarkable. Cerebrospinal fluid (CSF) analysis revealed elevated protein $(0.70 \mathrm{~g} / \mathrm{L})$ without pleocytosis. Electromyoneurography of lower extremities showed biphasic action potentials of very low amplitude without conduction delay. Somatosensory evoked potentials of posterior tibial and median nerves revealed prolonged conduction latencies at the thalamo-cortical level. Electroencephalogram comprised diffuse slow spikes-waves and focal slow activity over left frontocentrotemporal region interrupting background alpha rhythm. Brain magnetic resonance imaging (MRI) revealed multiple T2-weighted hyperintense lesions in bilateral frontoparietal subcortical white matter, predominantly on the left side (Figure), some of which were hyperintense on diffusion weighted images. Tests for antinuclear antibodies, anti-neutrophil cytoplasmic antibodies, paraneo-plastic antibodies and hepatitis antibodies were negative. Serum anti-ganglioside antibody assay demonstrated borderline values of anti-asialo GM1 antibody. Serology and CSF analysis for cytomegalovirus, Epstein-Barr virus, herpes simplex virus type 1 , varicella-zoster virus were all negative. Serological test for Borrelia burgdorferi, which was performed

From the Department of Neurology, General Hospital Dr. Josip Benčević, Slavonski Brod, Croatia.

Received November 11, 2013. Final Revisions Submitted December 10, 2013. Correspondence to: Bojan Rugole, Department of Neurology, General Hospital Dr. Josip Benčević, Andrije Štampara 42, HR-35000 Slavonski Brod, Croatia.

Email: rugole.b@gmail.com. 


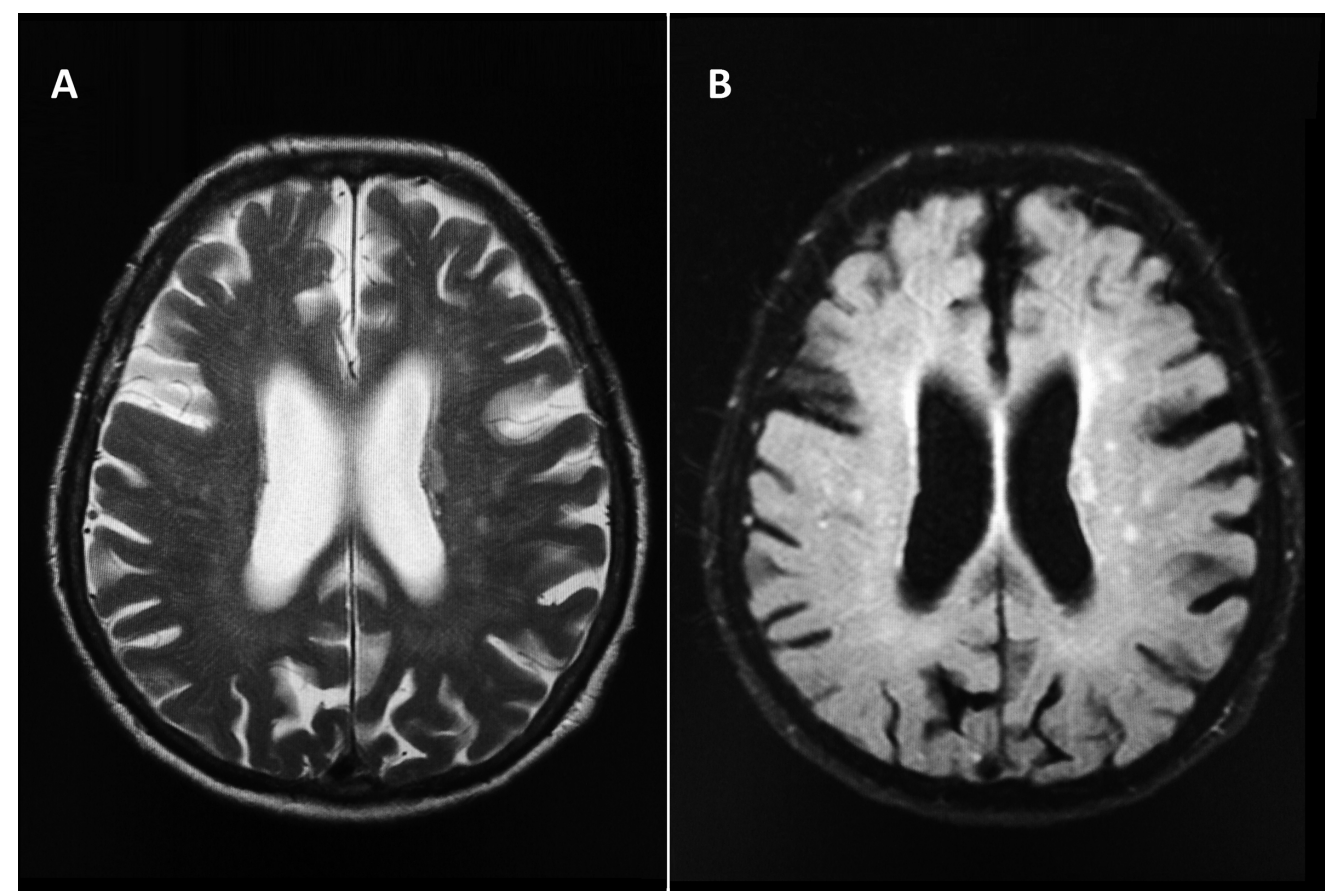

Figure: Axial T2-weighted (A) and fluid-attenuated inversion recovery (B) MRI images obtained 40 days after vaccination demonstrating hyperintensive white matter lesions

four weeks after first symptom onset, yielded positive results for $\operatorname{IgM}(31 \mathrm{AU} / \mathrm{mL})$ and negative for IgG. Since repeated tests for $\mathrm{IgG}$ came up negative this finding was interpreted as falsely positive.

Having been diagnosed with ADEM and AMAN, the patient was treated with methylprednisolone $250 \mathrm{mg} / \mathrm{day}$ intravenously for five days and subsequent oral prednisone taper for four weeks. Patient's condition improved within several days of treatment but paraparesis with diminished patellar reflexes persisted. Three months after intensive rehabilitation the patient was ambulatory again and started regaining enough strength to walk with minimal assistance.

\section{Discussion}

Most cases of ADEM and GBS are triggered by an infection. ${ }^{1,2}$ While it can appear in any age, ADEM is rare in adults and usually presents with slow-onset symptoms, sometimes weeks after preceding event. Lack of febrile response in elderly patients may mislead diagnosis. The disease typically features encephalopathy and focal neurologic signs with corresponding MRI findings of focal or multifocal white matter lesions. The most common form of GBS is AIDP, while AMAN accounts for less than $5 \%$ of GBS in Western countries. ${ }^{4}$ Symptoms of AMAN can evolve several weeks after initial event, comprising of dominantly proximal motor weakness with diminished reflexes without sensory symptoms, while electromyography shows diminished motor potential unit activity.

Neurologic complications following vaccination occur rarely, typically within a few months of vaccination. Vaccines which are usually reported to trigger autoimmune event include those against measles/mumps/rubella, diphtheria/tetanus/pertussis, rabies, smallpox, influenza, hepatitis A and B. ${ }^{3}$

The basic pathological mechanism in these disorders is thought to be an abnormal immune response to foreign antigens. In theory, viral antigens that share structural similarities with neural self-antigens induce autoimmunity through the activation of T or B cells, which leads to inflammatory response and tissue destruction. The existing evidence suggests that the autoimmune response is directed against the myelin sheath in both ADEM and AIDP. ${ }^{2,3}$ However, in case of the axonal form of GBS, autoimmune attack is directed against the axoplasm of peripheral nerves. ${ }^{2}$ Antibodies against GM1, GM1b and GD1a are frequently found in motor neuropathies. ${ }^{4}$

Simultaneous inflammation of both the central and peripheral nervous systems is seldom described in case reports as a complication following vaccination. It remains unclear why some individuals are susceptible to such an aberrant immunologic response. It can be debated that a combination of immunologic constitution, environmental factors and nature of the antigen stimulus determine threshold for such a response. The patient described in this case had symptoms of leg weakness following prior influenza vaccinations but, nevertheless, that was never considered to be an issue previously nor suspected of being an adverse reaction to vaccines. However, during this episode of illness no other disease or triggering event except vaccination was discovered, despite extensive diagnostic evaluations. Because of the low incidence of such cases and despite several studies which tried to address this connection, the existing evidence is, according to some authors, still inadequate 
to confirm or discard a causal relationship between influenza vaccine and ADEM and/or GBS..$^{5}$ In this case we were also unable to find a clear correlation between influenza vaccinations and described neurological complications. Nevertheless, the temporal association between those events is intriguing.

Both ADEM and AMAN usually respond well to corticosteroid treatment but additional treatment such as intravenous immunoglobulin or plasmapheresis is sometimes required. ${ }^{1,2}$ In comparison with those with AIDP, some patients with AMAN recover slower and require long term rehabilitation.

\section{Conclusion}

In adult patients who present with otherwise unexplained signs of encephalopathy or GBS, upon the exclusion of other possible causes, postvaccination etiology should also be suspected. Because of the low incidence of such complications it is still unknown which individuals are more susceptible to develop them. Given the lack of clear association with vaccines further investigations are warranted.

\section{REFERENCES}

1. Bennetto L, Scolding N. Inflammatory/post-infectious encephalomyelitis. J Neurol Neurosurg Psychiatry. 2004;75 (Suppl 1):i22-8.

2. Yuki N, Hartung HP. Guillain-Barré syndrome. N Engl J Med. 2012;366(24):2294-304.

3. Huynh W, Cordato DJ, Kehdi E, Masters LT, Dedousis C. Postvaccination encephalomyelitis: literature review and illustrative case. J Clin Neurosc. 2008;15:1315-22.

4. Hughes RA, Cornblath DR. Guillain-Barré syndrome. Lancet. 2005;366(9497):1653-6.

5. Stratton K, Ford A, Rusch E, Clayton EW, editors. Adverse effects of vaccines: evidence and causality. Committee to review adverse effects of vaccines. Washington D.C.: National Academies Press; 2011. p. 308-34. 